\title{
Chirp coded ultrasonic pulses used for scanning acoustic microscopy
}

\author{
Anowarul Habib and Frank Melandsø \\ anowarul.habib@uit.no \\ Department of Physics and Technology, UiT The Artic University of Norway, Norway
}

\begin{abstract}
In the present study, chirp coded pulses are investigated with respect to high frequency imaging. These pulses were used drive broadband polymer transducers assembled from an adhesive-free layer-be-layer deposition method. The image quality obtained from using a long chirp coded pulse was compared with images generated from shorter pulses. This includes the Ricker wavelet and the square pulse most commonly used for scanning acoustic microscopes.
\end{abstract}

Keywords: piezoelectric, polyvinylidenefluoride, Ricker wavelet, chirp signal, acoustic imaging.

\section{INTRODUCTION}

In high frequency acoustic imaging, the image quality depends on a number of factors like noise level, resolution, and penetration depth. There is always a tradeoff between resolution and penetration depth, where one event can be improved only at the expense of the other. By employing long coded sequences e.g. like chirp waves it has been possible to highly increase the signal to noise ratio (SNR). The increased SNR has made it possible to image deeper and at the same time obtain good resolution [1]. Chirp codes have commonly been employed for more than 50 years in many types of ultrasound applications including medical imaging, color flow imaging, synthetic aperture imaging, and non-destructive evaluation [27].

In acoustic imaging, the SNR also plays a vital role for the overall image quality. Coded excitation inherently possesses superior penetration and SNR compared to single square pulses that are most commonly used in acoustic imaging. This is due to the increased total wave energy per firing imposed by the longer coded sequences. However, if the depth resolution should be maintained, it is necessary to apply codes that are robust against wave distortion effects in the considered medium. The depth resolution also strongly relies upon the compression method used for the coded sequence.

Conventional scanning acoustic microscopy (SAM) uses high frequency acoustic waves that propagates inside the sample and reflects back with different velocities according to the stiffness of the tested sample with a high spatial resolution [8, 9]. For a typical SAM system the ultrasonic waves are excited through a piezoelectric transducer by applying a short high voltage square driving pulse. Single short pulses are very attractive from an electronics point of view, since they can be easily generated using fast high voltage switch/transistor circuits. However, their main drawback is the limited amount of energy in a single pulse, typically requiring high voltages in combination with averaging over many pulse firings to image objects with low acoustical contrast.

Conventional high frequency transducers are made from ceramic, single crystal, or piezoelectric thin films $(\mathrm{ZnO})$. The acoustic energy is typically focused using a concave spherical sapphire lens rod [10]. The main challenge with such design is the impedance mismatch between the refractive lens material (e.g., sapphire) and water, yielding for example, reduced sound transmission, bandwidth reduction, and geometrical aberration of the focusing beam [11]. Ferroelectric polymers like polyvinylidenefluoride (PVDF) and its copolymer with trifluoroethylene $[\mathrm{P}(\mathrm{VDF}-\mathrm{TrFE})]$ are widely used in sensor and transducer applications. Good acoustic match to many materials (e.g. water, polymers, and human tissue) and high chemical stability make these materials suitable for ultrasonic applications, especially in the high frequency end $(20 \mathrm{MHz}-1$ $\mathrm{GHz}$ ). The flexible film of PVDF and its copolymer fabricated by techniques such as spin coating, hot pressing, stamping and spraying on flat substrate [12-18].

In order to overcome the bandwidth issue related in chirp imaging, we here propose using a polymer based transducer for the acoustic imaging. Polymer based piezoelectric materials like PVDF and P(VDF-TrFE) are very suitable for achieving high frequencies and large bandwidths for ultrasonic sensors and transducers as shown in several applications [19-26]. The aim of this work has therefore been to investigate experimentally how longer chirped pulses can be used to improve the image quality in SAM systems.

\section{TRANSDUCER FABRICATIOIN}

A detailed fabrication process of the focused transducer has been published earlier by our group [25]. The proposed transducer design uses a layer-by-layer assembly of the piezoelectric copolymer $\mathrm{P}(\mathrm{VDF}-\mathrm{TrFE})$ and electrodes on top of a polyethyleneimines (PEI) polymer substrate. Before the assemble steps the substrate was prepared as an ultrasonic lens. This was achieved by milling a spherical shaped cavity into the counter-facing substrate side as shown in Fig. 1 as a two- 
dimensional side view. The combined substrate and lens material PEI was chosen due to its very good thermal stability, good impedance match to $\mathrm{P}(\mathrm{VDF}-\mathrm{TrFE})$ and very low acoustic attenuation [17].

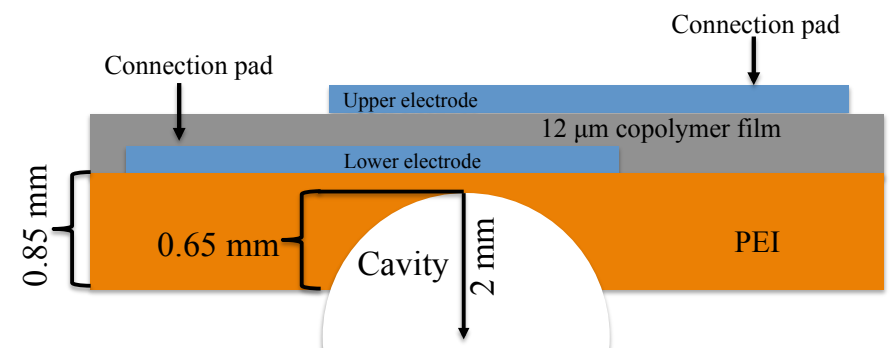

Fig. 1. A side view illustration of the various layers used in the transducer prototype.

As illustrated in Fig. 1, the tip of the spherical shaped milling cutter with diameter $2.0 \mathrm{~mm}$ was drilled $0.65 \mathrm{~mm}$ into the material to produce the spherical shaped lens cavity. These dimensions yielded a focal point around $2.5 \mathrm{~mm}$ from the lower PEI surface. After the milling, the spherical cavities were cleaned with acetone, ethanol, and dried with pressurized nitrogen to remove drill lubricant and polymer burrs.

For the following transducer assemble, the first step includes patterning of the lower electrode sitting on the PEI substrate. This patterning was done from a sequence of plasma sputtering through a high-resolution mask to produce a three-layer $(\mathrm{Cr} / \mathrm{Au} / \mathrm{Cr})$ electrode. Here $\mathrm{Cr}$ was chosen to yield good bonding to materials on both sides of the electrode. Then P(VDF-TrFE) in the fluid phase was spin coated on top of the lower electrode and thereafter dried to remove the solvent. A dried $\mathrm{P}(\mathrm{VDF}-\mathrm{TrFE})$ thickness around $12 \mu \mathrm{m}$ was achieved by adjusting the spin-coating parameters. Finally a three-layer $(\mathrm{Cr} / \mathrm{Au} / \mathrm{Cr})$ upper electrode was sputtered on top of the dried $\mathrm{P}(\mathrm{VDF}-\mathrm{TrFE})$ layer through another high resolution mask, which completed the transducer assembly process.

To pole and characterize the acoustic response, each of the transducers in the substrate were connected directly onto a PCB using spring contacts. Since these spring contacts yield open electrical lines, it was important to use small contacts to minimize additional inductive and capacities effects induced for the high frequency characterization. In order to make the P(VDF-TrFE) layer piezoelectric, it was polarized at room temperature by connecting a high voltage AC source to the lower electrodes, while the upper ones were grounded. Further details regarding transducer production, annealing, and poling steps can be found in Ref [27].

\section{EXPERIMENTAL PROCEDURE}

To investigate the effects of using chirp-coded waveforms, several in-house transducer prototypes were built and inserted in a three-dimension (3D) scanning system to generate images. A schematic diagram of the scanning system is shown in Fig. 2. Acoustic signals were generated using a signal generator (Agilent 81150A) and delivered to the transducer.

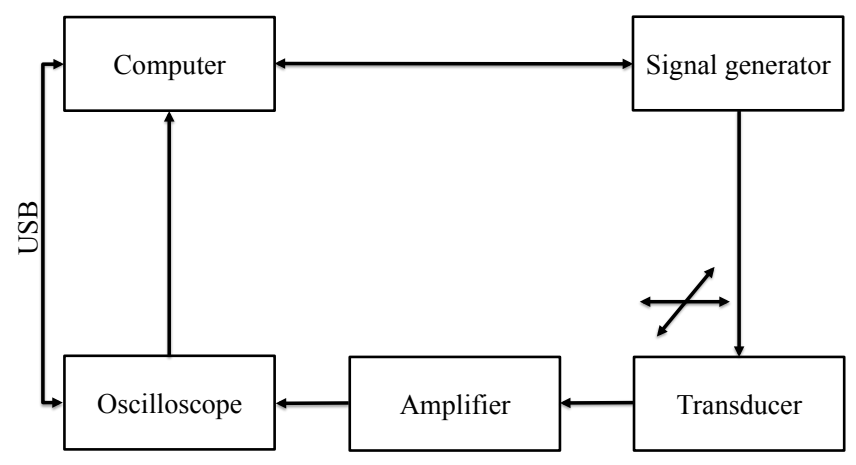

Fig. 2. Schematic diagram for experimental set up used to image surface of the test object.

The reflected signals from the surface of the coin picked up by the transducer were fed into a trans-impedance current amplifier (FEMTO DHPCA-100), which converts the current into an output potential. Finally, the output voltage from this amplifier was digitized by an oscilloscope (Agilent 3024A). This oscilloscope performs averaging over 256 pulse shootings, digitize the signal, and load it into a personal computer (PC) over an USB connection. The PC with designated software was employed to record the time domain signal for each particular pixel and convert them into an image.

For comparison, images were also generated from driving the transducer with shorter broadband pulses, like the Ricker wavelet, and the square pulse most commonly used for SAM. These waveforms were downloaded into an arbitrary signal generator (Agilent 81150A) capable of producing output signals up to 10 Volts peak to peak.

In order to generate images the transducer was scanned over a two of the axis (denoted as the XY plane) with predefined steps in the $\mathrm{X}$ and $\mathrm{Y}$ directions.

\section{RESULTS AND DISCUSSIONS}

To explore the effect of using different pulses to excite the polymer transducers, the three different waveforms shown in Fig. 3 were applied. These are the square pulse, the Ricker wavelet and the chirp code with time domain signals shown in Figs. (a), (c), and (e), respectively. The corresponding Fourier spectra are shown in Figs. (b), (d), and (f).

We have noticed from the spectra in Fig. 3 that the Ricker and chirp pulses both have their maximum energy around $40 \mathrm{MHz}$. This frequency will be in the vicinity of the center frequency of the used transducer prototype with a dried P(VDF-TrFE) thickness around $12 \mu \mathrm{m}$. From Fig. 3 (b) we also observe that the square pulse has substantial energy at 40 $\mathrm{MHz}$, allowing us to compare the backscattered echoes using these three waveforms as driving pulses. 

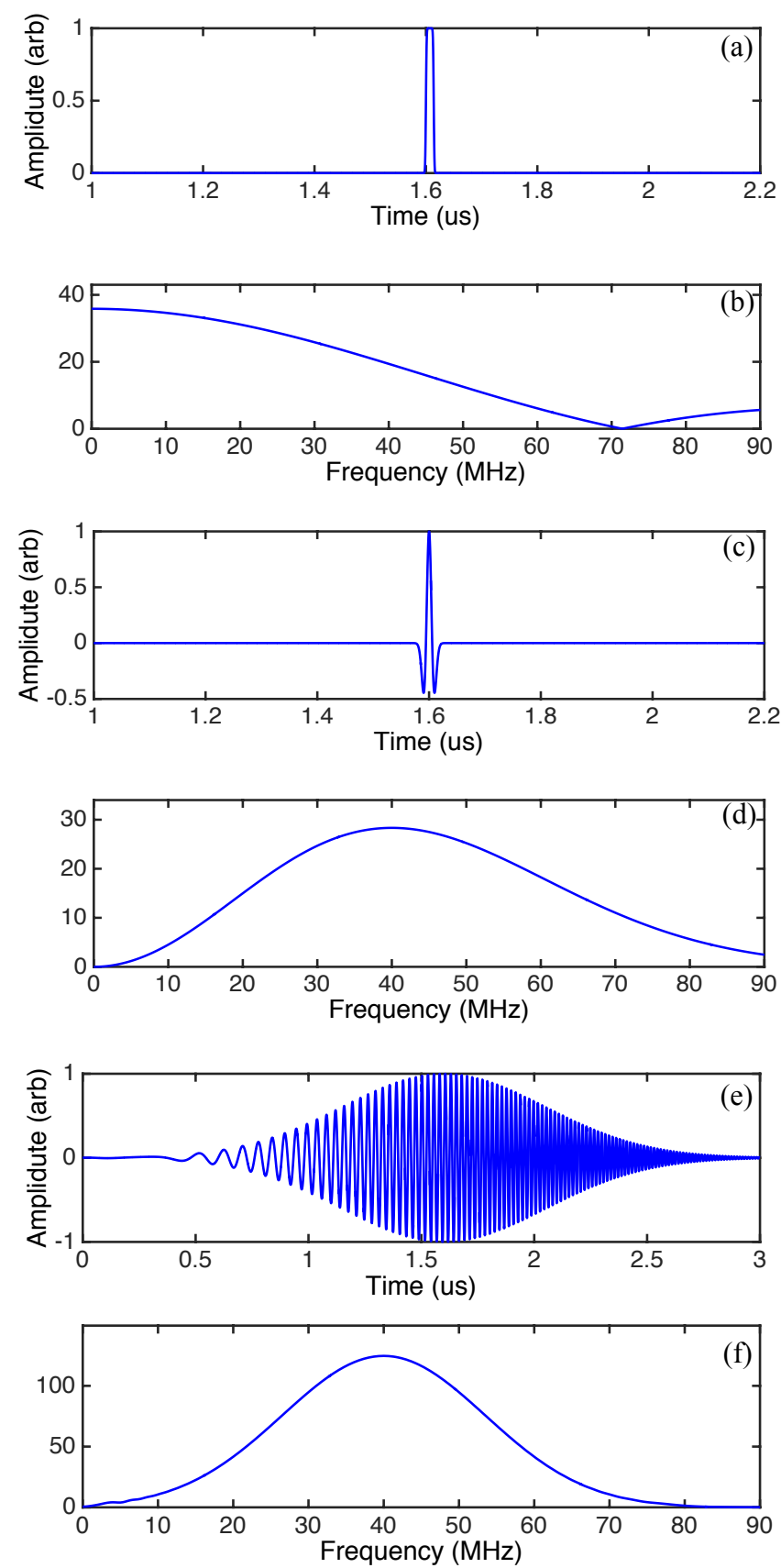

Fig. 3: Different waveforms used in the investigation including the square pulse shown as a function of time in Fig (a) and amplitude spectrum shown in Fig. (b). The corresponding plots for the Ricker wavelet and the chirp signal are shown in Figs. (c), (d) and (e), (f), respectively.

To explore how these three waveforms perform in an imaging system, one of the transducer prototypes was mounted in a water tank and scanned in a two-dimensional (2D) plane over a test object as previously described. As a test object, a selected area with size $8.4 \mathrm{~mm} \times 4.8 \mathrm{~mm}$ of a coin (Norwegian 1 krone) was chosen. An optical image of the coin is shown in Fig. 4 (a) with the ultrasonic scanning area indicated. The images produced from a coin surface from the scanning are shown in Fig. 4. Figures 4 (b), (c), and (d) show the received images when the transducer was excited with the Ricker wavelet, square pulse, and chirp signal, respectively. The transducer was focused at the coin surface, and the voltage amplitudes from the received echo were plotted for each step, to create $2 \mathrm{D}$ images. The specified step size $(50 \mu \mathrm{m}$ in both $\mathrm{X}$ and $\mathrm{Y}$-direction) will then provide the physical pixel size.

From Fig. 4 we notice that all waveforms yielded images with comparable resolutions. However, there is a substantial difference in the noise levels, where the image generated from the chirped signal [Fig. 4 (d)] having the largest SNR. We also see that Fig. 4 (b) yields a larger SNR than (c). The observed SNR values are all consistent with the total energies of the input pulses around the transducers center frequency at 40 $\mathrm{MHz}$, as previously shown by the frequency spectra in Fig. 3 . For Figs. 4 (c) and (d) there are also a few noticeable artifacts with circular shape, probably occurring from bubbles sitting on the coin surface.
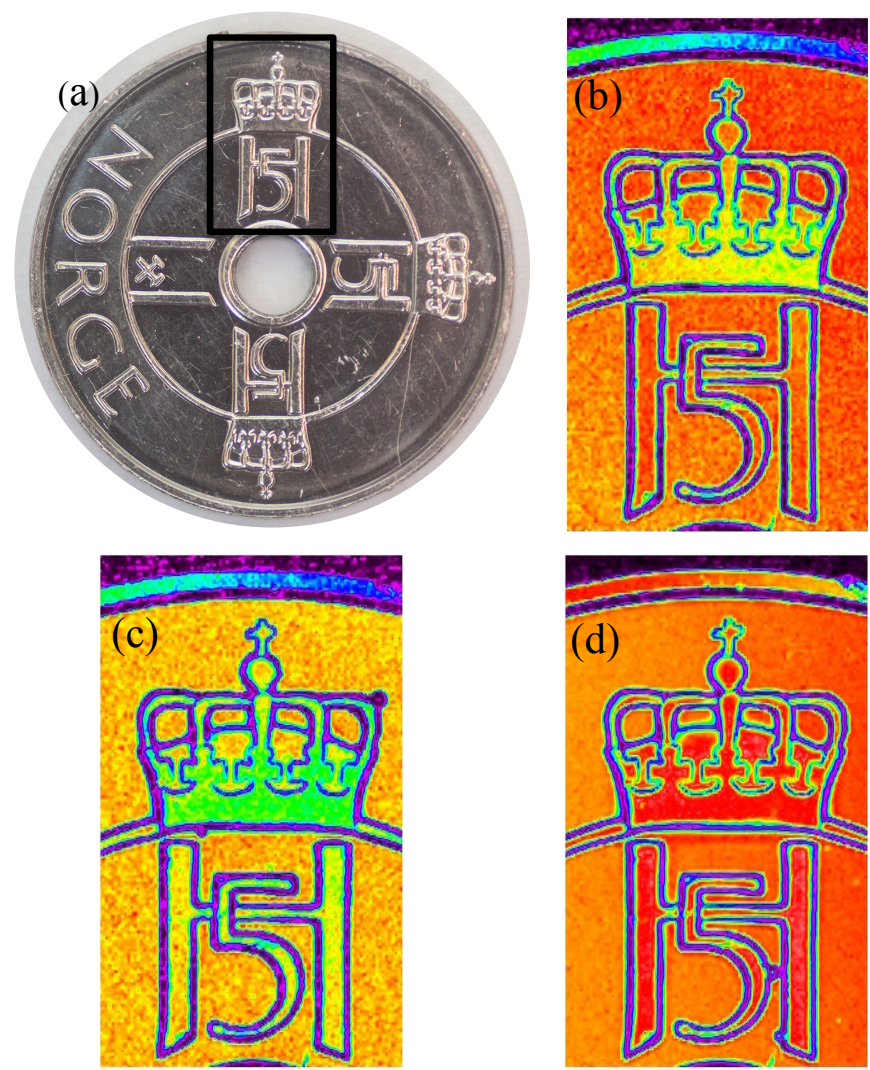

Fig. 4. Optical image of the coin used as the test object [Fig. (a)] and ultrasonic amplitude images within the indicated scanning area, using a Ricker wavelet [Fig (b)], square wave [Fig. (c)], and chirp wave [Fig (d)] as the driving pulse. The selected scanning area was $8.4 \mathrm{~mm} \times 4.8 \mathrm{~mm}$ with a $50 \mu \mathrm{m}$ step size in both directions.

\section{CONCLUSION}

In this study, we have investigated the capability of using adhesive-free $\mathrm{P}(\mathrm{VDF}-\mathrm{TrFE})$ transducers for high frequency imaging, and how these transducers perform using different 
driving pulses. Our investigation shows that several pulses, including square pulses, Ricker wavelets, and chip pulses, all are suitable for these transducers due to their large bandwidth. Image tests performed on a coin also indicates that the longer chirped pulse has advantages in terms of producing images with a higher SNR. However, since the reported tests are only preliminary, it is necessary to explore the capability of these

\section{ACKNOWLEDGEMENT}

This work was supported by The Research Council of Norway through the project "Subsea sensors".

\section{References}

[1] T. Misaridis and J. A. Jensen, "Use of modulated excitation signals in medical ultrasound. Part II: Design and performance for medica imaging applications," IEEE transactions on ultrasonics, ferroelectrics, and frequency control, vol. 52, pp. 192-207, 2005.

[2] F. Zhao, L. Tong, Q. He, and J. Luo, "Coded excitation for diverging wave cardiac imaging: a feasibility study," Phys. Med. Biol., vol. 62, p. $1565,2017$.

[3] T. L. Szabo, Diagnostic ultrasound imaging: inside out: Academic Press, 2004

[4] G. S. Kino, Acoustic waves: devices, imaging and analog signal processing, 1987.

[5] H. Zhao, L. Y. Mo, and S. Gao, "Barker-coded ultrasound color flow imaging: Theoretical and practical design considerations," IEEE Trans. Ultrason. Ferroelectr. Freq. Control., vol. 54, 2007.

[6] D. J. Muzilla, R. Y. Chiao, and A. L. Hall, "Method and apparatus for color flow imaging using coded excitation with single codes," ed: Google Patents, 1999.

[7] R. Y. Chiao, "Method and apparatus for flow imaging using Golay codes," ed: Google Patents, 2001.

[8] A. Habib, A. Shelke, M. Vogel, S. Brand, X. Jiang, U. Pietsch, et al., "Quantitative Ultrasonic Characterization of c-axis oriented polycrystalline AIN thin film for smart device application," Acta Acust united Ac., vol. 101, pp. 675-683, 2015.

[9] A. Habib, A. Shelke, M. Vogel, U. Pietsch, X. Jiang, and T. Kundu, "Mechanical characterization of sintered piezo-electric ceramic material using scanning acoustic microscope," Ultrasonics, vol. 52, pp. 989-995, 2012.

[10] W. Zou, S. Holland, K. Y. Kim, and W. Sachse, "Wideband highfrequency line-focus PVDF transducer for materials characterization," Ultrasonics, vol. 41, pp. 157-161, 2003.

[11] S. Smolorz and W. Grill, "Focusing PVDF transducers for acoustic microscopy," Res. Nondestr. Eval., vol. 7, pp. 195-201, 1996.

[12] K. Kimura and H. Ohigashi, "Generation of very high-frequency ultrasonic waves using thin films of vinylidene fluoride-trifluoroethylene copolymer," J. Appl. Phys., vol. 61, pp. 4749-4754, 1987.

[13] M. Robert, G. Molingou, K. Snook, J. Cannata, and K. K. Shung, "Fabrication of focused poly (vinylidene fluoride-trifluoroethylene) P (VDF-TrFE) copolymer 40-50 MHz ultrasound transducers on curved surfaces," J. Appl. Phys., vol. 96, pp. 252-256, 2004.

[14] J. S. Jeong and K. K. Shung, "Improved fabrication of focused single element P (VDF-TrFE) transducer for high frequency ultrasound applications," Ultrasonics, vol. 53,pp. 455-458, 2013. transducers further. This should be done for instance, by repeating the tests such as on biological samples yielding $3 \mathrm{D}$ structures with much weaker backscattering. It is also important to explore further how the length of the coded pulses and different compression methods will influence the image quality.

[15] L. F. Brown and D. L. Carlson, "Ultrasound transducer models for piezoelectric polymer films," IEEE Trans. Ultrason. Ferroelectr. Freq. Control., vol. 36, pp. 313-318, 1989

[16] L. Brown, R. Carlson, and J. Sempsrott, "Spin-cast P (VDF-TrFE) films for high performance medical ultrasound transducers," in Ultrasonics Symposium, 1997. Proceedings., 1997 IEEE, 1997, pp. 1725-1727.

[17] A. Decharat, S. Wagle, and F. Melandso, "Evaluation of the acoustical properties of adhesive-free dual layer piezoelectric PVDF copolymer transducer," in European Frequency and Time Forum \& International Frequency Control Symposium (EFTF/IFC), 2013 Joint, 2013, pp. 266269.

[18] C.-H. Chung and Y.-C. Lee, "Fabrication of poly (vinylidene fluoridetrifluoroethylene) ultrasound focusing transducers and measurements of elastic constants of thin plates," NDT \& E International, vol. 43, pp. 96105,2010 .

[19] P. E. Bloomfield, W.-J. Lo, and P. A. Lewin, "Experimental study of the acoustical properties of polymers utilized to construct PVDF ultrasonic transducers and the acousto-electric properties of PVDF and $\mathrm{P}$ (VDF/TrFE) films," IEEE Trans. Ultrason. Ferroelectr. Freq. Control. vol. 47, pp. 1397-1405, 2000

[20] M.-K. Chae, M.-J. Kim, K.-L. Ha, and C.-B. Lee, "Focal length controllable ultrasonic transducer using bimorph-type bending actuator," Jpn. J. Appl. Phys., vol. 42, p. 3091, 2003.

[21] L. Xi, X. Li, and H. Jiang, "Variable-thickness multilayered polyvinylidene fluoride transducer with improved sensitivity and bandwidth for photoacoustic imaging," Appl. Phys. Lett., vol. 101, p. $173702,2012$.

[22] F. Melandsø, S. Wagle, A. Decharat, A. Habib, and B. S. Ahluwalia, "High-frequency poly (vinylidene fluoride) copolymer transducers used for spectral characterization of settled microparticles," Jpn. J. Appl. Phys., vol. 55, p. 07KB07, 2016.

[23] A. Decharat, S. Wagle, and F. Melandsø, "Effect of polymer electrode thickness on the acoustical properties of all-screen printed piezoelectric pvdf copolymer transducers," Jpn. J. Appl. Phys., vol. 53, p. 05HB16, 2014.

[24] S. Wagle, A. Decharat, A. Habib, B. S. Ahluwalia, and F. Melandsø, "Evaluation of adhesive-free crossed-electrode poly (vinylidene fluoride) copolymer array transducers for high frequency imaging," Jpn. J. Appl. Phys., vol. 55, p. 07KE11, 2016.

[25] S. Wagle, A. Habib, and F. Melandsø, "Ultrasonic measurements of surface defects on flexible circuits using high-frequency focused polymer transducers," Jpn. J. Appl. Phys, vol. 56, p. 07JC05, 2017.

[26] S. Wagle, A. Habib, and F. Melandsø, "Temperature influence on high frequency copolymer transducers," in IEEE Int. Ultrason. Symp., 2016, pp. 1-4.

[27] S. Wagle, A. Decharat, and F. Melandsø, "Adhesive-free dual layer piezoelectric PVDF copolymer transducers in sender and receiver sequences," in IEEE Int. Ultrason. Symp., 2014, pp. 2063-2066. 